\title{
A Generic Non-Stationary MIMO Channel Model for Different High-Speed Train Scenarios
}

\author{
Ammar Ghazal ${ }^{1}$, Cheng-Xiang Wang ${ }^{1}$, Yu Liu ${ }^{2}$, Pingzhi Fan ${ }^{3}$, Mohamed Khaled Chahine ${ }^{4}$ \\ ${ }^{1}$ Institute of Sensors, Signals and System, Heriot-Watt University, Edinburgh EH14 4AS, UK. \\ ${ }^{2}$ School of Information Science and Engineering, Shandong University, Shandong 250100, China. \\ ${ }^{3}$ Institute of Mobile Communications, Southwest Jiaotong University, Chengdu, Sichuan 610031, China. \\ ${ }^{4}$ Department of Electronics and Communications Engineering, FMEE, Damascus University, Damascus, Syria. \\ Email: \{ag289, cheng-xiang.wang\}@hw.ac.uk, xinwenliuyu@163.com, p.fan@ieee.org, mkchahine@gmail.com
}

\begin{abstract}
This paper proposes a generic non-stationary wideband geometry-based stochastic model (GBSM) for multipleinput multiple-output (MIMO) high-speed train (HST) channels. The proposed generic model can be applied on the three most common HST scenarios, i.e., open space, viaduct, and cutting scenarios. A good agreement between the statistical properties of the proposed generic model and those of relevant measurement data from the aforementioned scenarios demonstrates the utility of the proposed channel model.
\end{abstract}

Keywords-High-speed train (HST) channels, high-speed railway scenarios, GBSM, non-stationary MIMO channel models, open space, viaduct, cutting.

\section{INTRODUCTION}

High-mobility scenarios, e.g., HST scenarios, are expected to be typical scenarios for fifth generation $(5 \mathrm{G})$ communication systems [1]. With the rapid development of HSTs, an increasing volume of wireless communication data is required to be transferred to train passengers. HST users demand high network capacity and reliable communication services regardless of their locations or speeds. To satisfy these demands, HST wireless communication systems have to overcome many challenges resulting from the high speed of the train that can easily exceed $250 \mathrm{~km} / \mathrm{h}$, such as fast handover, fast travel through widely diverse scenarios, and large Doppler spreads [2] besides some challenges inherited from conventional trains such as high penetration losses, limited visibility in tunnels, and the harsh electromagnetic environment [3].

The transmitter (Tx) and receiver ( $\mathrm{Rx})$ of a HST wireless communication system encounter different channel conditions due to the difference of surrounding geographical environments. The HST environment can be generally classified into the following main scenarios: open space, viaduct, cutting, hilly terrain, tunnels, and stations. Considering some unique setup of the aforementioned scenarios and some other special HST scenarios, HST environment can be further classified into 12 scenarios [4]. HST can operate across one or more of these scenarios during its travel. The propagation characteristics change significantly with the change of environments and the distance between the $\mathrm{Tx}$ and $\mathrm{Rx}$ even in the same terrain. Scenarios have close relationship with channel modeling and measurements. Most standard channel models in the literature, like UMTS, COST 2100, and IMT-2000 failed to introduce any of the HST scenarios. The moving networks scenario in the WINNER II channel model [5] and rural macro-cell (RMa) scenario in the IMT-A channel model [6] have only considered a rural environment for HSTs, while neglecting other HST scenarios. This has motivated us to fill in the gap in the literature by proposing a novel generic HST channel model that can be applied on different HST scenarios. The proposed generic channel model is an extension of the one proposed in [7] for open space scenario. The extension includes introducing scenario-specific time-variant Ricean $K$-factor and time-variant distance between $\mathrm{Tx}$ and $\mathrm{Rx}$ that take into account key parameters of the considered scenarios. The proposed model will be applicable on three of the most common HST scenarios, i.e, open space, viaduct, and cutting scenarios. For example, these three scenarios form around $84 \%$ of the HST scenarios that can be encountered along the Zhengzhou-Xian HST line in China [8]. It is noteworthy that this generic model can be further applied on other HST scenarios by choosing proper values of some of its key parameters.

The rest of this paper is organized as follows. The three most common HST scenarios, i.e., open space, viaduct, and cutting, are introduced in Section II. In Section III, a generic GBSM for MIMO HST channels in the aforementioned scenarios is proposed. To verify the proposed generic model using real measured data, relevant statistical properties are derived in Section IV. The simulation results and the analysis are presented in Section V. Finally, the conclusions are drawn in Section VI.

\section{HST SCENARIOS}

\section{A. Open space}

In the open space scenario, also called plain scenario, the HST is moving at a very high speed in a rural area where the BS antenna is much higher than the surroundings. This environment focuses on large cells and continuous coverage where the link between the fixed BS and the moving terminal normally has a dominant line-of-sight (LoS) component. Based on the geographic nature and the distribution / height of the surrounding scatterers, the open scenarios can be further classified into rural, urban, and suburban.

\section{B. Viaduct}

The viaduct scenario is common for HSTs, e.g., viaducts form $86.5 \%$ of Beijing-Shanghai HST railway line. The main purpose of viaducts is to ensure the smoothness of the rail, high speed of the train. In this scenario, the radio reflection, scattering, and diffraction caused by nearby scatterers, e.g., 
trees and buildings, can also be reduced significantly. The viaduct height and relative $\mathrm{BS}$ height have great influence on the received signal. Because of the relatively high altitude of the viaduct in comparison with the surrounding terrain, the LoS component is dominant in this scenario. However, the sparsity of the scatterers in the environment around the viaduct will still influence the received signal at the Rx. Based on the relative altitude between the scatterers and the viaduct, this scenario can be further classified into a high viaduct scenario, where most scatterers located nearby the viaduct are lower than the surface of the viaduct and therefore their impact on the propagation characteristics is negligible, and a low viaduct scenario, where some of the nearby scatterers are higher than the surface of the viaduct and consequently they introduce rich reflections and scattering components that may result in a severe shadow fading and / or extra path loss [4].

\section{Cutting}

The cutting scenario is another common scenario for HST wireless communications. It represents an environment where the HST passes a U-shaped geographical cut surface between the hills. The cutting is widely used for HST construction to ensure the smoothness of the rail and help to achieve a high speed of the train when passing through hills. The propagation of radio waveforms in this scenario is significantly affected by the steep walls on both sides. The LoS component can be observed along the route of the HST in this scenario. Here, we can identify two cutting scenarios: deep cutting if the antenna mounted on top of the train is lower than the upper eave of the cutting and low cutting if the height of the upper eave is lower than the top of the antenna of the train.

\section{A GENERIC MODEL FOR HST CHANNELS}

We consider an HST communication system that adopts the IMT-A cellular network architecture where mobile relay stations (MRSs) are deployed on the surface of the train. As a result, the end-to-end communications between the BS and MS will consist of two channels: outdoor channel and indoor one. Here, we will focus on the outdoor channel between the BS and MRS in the different HST scenarios. A MIMO HST system is considered with $S$ transmit and $U$ receive omni-directional antenna elements. The BS is located on the track-side with the minimum distance between the BS and the mid of the track denoted as $D_{\text {min }}$. The time-varying distance between the BS and MRS is $D_{s}(t)=\sqrt{\left(D_{\text {proj }}^{2}(t)+h^{2}\right)}$, where $h$ is the relative height between BS and MRS, and $D_{\text {proj }}(t)=\sqrt{\left(D_{\text {min }}^{2}+D_{\mathrm{ver}}^{2}(t)\right)}$ is the projection of $D_{s}(t)$ on the railway track plane. The distance $D_{\text {ver }}(t)$ stands for the vertical distance between the bottom of the BS and the projection of the MRS location on the railway track plane. Fig. 1 illustrates the proposed GBSM, which consists of multiple confocal ellipses with single-bounced rays and the LoS component [7]. For clarity purposes, we use a $2 \times 2 \mathrm{MIMO}$ channel model in Fig. 1 as an example. The parameters in Fig. 1 are defined in Table I.

Based on the tapped delay line (TDL) structure, the taps are represented by multiple confocal ellipses with the BS and MRS located at the foci. There are $N_{i}$ effective scatterers on the $i$ th ellipse (i.e., $i$ th tap), where $i=1,2, \ldots, I$ and $I$ is the total number of ellipses or taps. Each effective scatterer is intended to represent the effect of many physical scatterers within the region. The semi-major axis of the $i$ th ellipse and the $n_{i}$ th $\left(n_{i}=\right.$ $\left.1, \ldots, N_{i}\right)$ effective scatterer are denoted by $a_{i}(t)$ and $s^{\left(n_{i}\right)}$, respectively. We denote the time-varying semi-minor axis of the $i$ th ellipse as $b_{i}(t)=\sqrt{a_{i}^{2}(t)-f_{s}^{2}(t)}$, where $f_{s}(t)=$ $D_{s}(t) / 2$ represents a half of the distance between the two foci of ellipses. The tilt angles of the BS and MRS antenna arrays are denoted by $\beta_{T}$ and $\beta_{R}$, respectively. The MRS moves with the same speed $v_{R}$ as the train in the direction determined by the angle of motion $\gamma_{R}$. The angle of arrival (AoA) of the wave traveling from an effective scatterer $s^{\left(n_{i}\right)}$ to the MRS is denoted by $\phi_{R}^{\left(n_{i}\right)}(t)$. The angle of departure (AoD) of the wave that impinges on the effective scatterer $s^{\left(n_{i}\right)}$ is denoted by $\phi_{T}^{\left(n_{i}\right)}(t)$, while $\phi_{T_{p}}^{\operatorname{LoS}}(t)$ denotes the AoA of a LoS path.

The complex channel impulse response between the $p$ th $(p=1, \ldots, S)$ element of the BS, $T_{p}$, and the $q$ th $(q=1, \ldots, U)$ element of the MRS, $R_{q}$, can be expressed as $h_{p q}(t, \tau)=$ $\sum_{i=1}^{I} h_{i, p q}(t) \delta\left(\tau-\tau_{i}\right)$, where $h_{i, p q}(t)$ and $\tau_{i}$ denote the complex space-time-variant tap coefficients and the discrete propagation delay of the $i$ th tap, respectively. From the above GBSM, the complex tap coefficients for the first tap $(i=1)$ of the $T_{p}-R_{q}$ link is a superposition of the LoS component and single-bounced (SB) components, and can be expressed as

$$
h_{1, p q}(t)=h_{1, p q}^{\mathrm{LoS}}(t)+h_{1, p q}^{\mathrm{SB}}(t)
$$

where

$h_{1, p q}^{\mathrm{LoS}}(t)=\sqrt{\frac{K_{p q}(t)}{K_{p q}(t)+1}} e^{-j 2 \pi f_{c} \tau_{p q}(t)} e^{j 2 \pi f_{\max } t \cos \left(\phi_{T_{p}}^{\mathrm{LoS}}(t)-\gamma_{R}\right)}$

$$
\begin{array}{r}
h_{1, p q}^{\mathrm{SB}}(t)=\sqrt{\frac{\Omega_{1, p q}}{K_{p q}(t)+1}} \sum_{n_{1}=1}^{N_{1}} \frac{1}{\sqrt{N_{1}}} e^{j\left(\psi_{n_{1}}-2 \pi f_{c} \tau_{p q, n_{1}}(t)\right)} \\
\times e^{j 2 \pi f_{\max } t \cos \left(\phi_{R}^{\left(n_{1}\right)}(t)-\gamma_{R}\right)} .
\end{array}
$$

The complex tap coefficients for other taps $(1<i \leq I)$ of the $T_{p}-R_{q}$ link is a sum of $\mathrm{SB}$ components only and can be expressed as

$$
\begin{aligned}
& h_{i, p q}(t)=h_{i, p q}^{\mathrm{SB}}(t)=\sqrt{\Omega_{i, p q}} \sum_{n_{i}=1}^{N_{i}} \frac{1}{\sqrt{N_{i}}} \\
& \times e^{j\left(\psi_{n_{i}}-2 \pi f_{c} \tau_{p q, n_{i}}(t)\right)} e^{j 2 \pi f_{\max } t \cos \left(\phi_{R}^{\left(n_{i}\right)}(t)-\gamma_{R}\right)}, 1<i \leq I .
\end{aligned}
$$

It is worth mentioning that in (2a), (2b), and (3), we have timevarying parameters $\tau_{p q}(t), \phi_{T_{p}}^{\mathrm{LoS}}(t), \tau_{p q, n_{i}}(t)(i=1, \ldots, I)$, and $\phi_{R}^{\left(n_{i}\right)}(t)$, which make the underlying GBSM a non-stationary one. If these parameters are not time-varying, then the GBSM can be reduced to a side-sense stationary (WSS) one.

In (2a), (2b), and (3), $\Omega_{i, p q}$ designates the mean power for the $i$ th tap, $\tau_{p q}(t)=\varepsilon_{p q}(t) / c$, and $\tau_{p q, n_{i}}(t)=\left(\varepsilon_{p n_{i}}(t)+\right.$ $\left.\varepsilon_{n_{i} q}(t)\right) / c$ are the travel times of the waves through the links $T_{p}-R_{q}$ and $T_{p}-s^{\left(n_{i}\right)}-R_{q}$, respectively, as shown in Fig. 1. Here, $c$ represents the speed of light and the symbol $K_{p q}(t)$ designates the Ricean factor. The phases $\psi_{n_{1}}$ and $\psi_{n_{i}}$ are independent and identically distributed (i.i.d.) random 
variables with uniform distributions over $[-\pi, \pi)$ and $f_{\max }$ is the maximum Doppler shift related to the MRS. From Fig. 1 and based on the law of cosines, we have [9]

$$
\begin{aligned}
& \varepsilon_{p q}(t) \approx D_{s}(t)-k_{p} \Delta x_{T} \cos \beta_{T}-k_{q} \Delta x_{R} \cos \left(\phi_{T_{p}}^{\operatorname{LoS}}(t)-\beta_{R}\right) \\
& \varepsilon_{p n_{i}}(t) \approx \xi_{T}^{\left(n_{i}\right)}(t)-k_{p} \Delta x_{T} \cos \left(\phi_{T}^{\left(n_{i}\right)}(t)-\beta_{T}\right) \\
& \varepsilon_{n_{i} q}(t) \approx \xi_{R}^{\left(n_{i}\right)}(t)-k_{q} \Delta x_{R} \cos \left(\phi_{R}^{\left(n_{i}\right)}(t)-\beta_{R}\right)
\end{aligned}
$$

where $k_{p}=(S-2 p+1) / 2, \quad k_{q}=(U-2 q+1) / 2$, and $\xi_{R}^{\left(n_{i}\right)}(t)=b_{i}^{2}(t) /\left(a_{i}(t)+f_{s}(t) \cos \phi_{R}^{\left(n_{i}\right)}(t)\right)$ with $\xi_{T}^{\left(n_{i}\right)}(t)=$ $\left(a_{i}^{2}(t)+f_{s}^{2}(t)+2 a_{i}(t) f_{s}(t) \cos \phi_{R}^{\left(n_{i}\right)}(t)\right) /\left(a_{i}(t)+f_{s}(t) \cos \phi_{R}^{\left(n_{i}\right)}(t)\right)$.

Note that the AoD $\phi_{T}^{\left(n_{i}\right)}(t)$ and AoA $\phi_{R}^{\left(n_{i}\right)}(t)$ are interdependent for SB rays. The relationship between the AoD and AoA for multiple confocal ellipses model can be given by [9]

$$
\sin \phi_{T}^{\left(n_{i}\right)}(t)=\frac{b_{i}^{2}(t) \sin \phi_{R}^{\left(n_{i}\right)}(t)}{a_{i}^{2}(t)+f_{s}^{2}(t)+2 a_{i}(t) f_{s}(t) \cos \phi_{R}^{\left(n_{i}\right)}(t)}
$$

The time-variant LoS AoA $\phi_{T_{p}}^{\operatorname{LoS}}(t)$ can be expressed as

$\phi_{T_{p}}^{\mathrm{LoS}}(t)=\left\{\begin{array}{l}\phi_{T_{p}}^{\mathrm{LoS}}\left(t_{0}\right)+\arccos \left(\frac{D_{s}\left(t_{0}\right)+v_{R} t \cos \gamma_{R}}{D_{s}(t)}\right),-\pi \leq \gamma_{R} \leq 0 \\ \phi_{T_{p}}^{\mathrm{LoS}}\left(t_{0}\right)-\arccos \left(\frac{D_{s}\left(t_{0}\right)+v_{R} t \cos \gamma_{R}}{D_{s}(t)}\right), \quad 0 \leq \gamma_{R} \leq \pi\end{array}\right.$

where $\phi_{T_{p}}^{\operatorname{LoS}}\left(t_{0}\right)=\arcsin \left(\frac{k_{p} \Delta x_{T}}{D_{s}\left(t_{0}\right)} \sin \beta_{T}\right)$ denotes the initial LoS AoA at time $t=t_{0}$.

Here, we use the von Mises PDF to describe the timevarying angles $\phi_{T}^{\left(n_{i}\right)}(t)$ and $\phi_{R}^{\left(n_{i}\right)}(t)$ as it is a general function and includes some well-known PDFs as special cases, e.g., uniform and Gaussian PDFs [9]. The von Mises PDF is defined as $f(\phi) \triangleq \exp [k \cos (\phi-\mu)] /\left[2 \pi I_{0}(k)\right]$, where $\mu$ is the mean value of angle $\phi \in[-\pi, \pi), I_{0}(\cdot)$ is the zeroth-order modified Bessel function of the first kind, and $k(k \geq 0)$ is a positive real-valued parameter that controls the spread of $\phi$. Applying the von Mises distribution to the time-varying AoAs, we get $f\left(\phi_{R}^{(i)}\right)(t) \triangleq \exp \left[k_{R}^{(i)} \cos \left(\phi_{R}^{(i)}-\mu_{R}^{(i)}(t)\right)\right] /\left[2 \pi I_{0}\left(k_{R}^{(i)}\right)\right]$, where $\mu_{R}^{(i)}$ is the mean angular value of the AoA $\phi_{R}^{(i)}$ and $k_{R}^{(i)}$ is the relevant von Mises parameter that controls the spread of $\phi_{R}^{(i)}$. Similarly, we can get $f\left(\phi_{T}^{(i)}\right)(t)$ with $\mu_{T}^{(i)}$ and $k_{T}^{(i)}$. By considering a non-isotropic scattering environment, we can use the modified method of equal areas (MMEA) to calculate the discrete AoAs $\left\{\phi_{R}^{\left(n_{i}\right)}\right\}_{n_{i}=1}^{N_{i}}$ that can be determined by finding the solutions of the following equation using numerical rootfinding techniques [9]:

$$
\frac{n_{i}-\frac{1}{4}}{N_{i}}-\int_{\mu_{R}^{(i)}\left(t_{0}\right)-\pi}^{\phi_{R}^{\left(n_{i}\right)}} f\left(\phi_{R}^{(i)}\right)(t) \mathrm{d} \phi_{R}^{(i)}=0, \quad n_{i}=1,2, \ldots, N_{i} .
$$

where reasonable values of $N_{i}$ are in the range of $40-50$.

The MRS is moving with the speed of $v_{R}$ in the direction defined by the angle of motion $\gamma_{R}$. Correspondingly, the AoAs and the axes of the ellipses will be changed. The time-varying function of mean AoA $\mu_{R}^{(i)}(t)$ can be expressed as (8) [7], which is presented at the top of the next page.

This model has been verified in open space scenario [7] by comparing its stationary interval with the one measured for a real HST channel and reported in [10]. In the following two subsections, we explain the extension of the model in viaduct and cutting scenarios before verifying this extension using measurement data from both scenarios in Section V.

\section{A. Viaduct}

Fig. 2 shows a sectional view of the viaduct scenario with the main parameters of the viaduct structure that influence the wireless signal received by the MRS mounted on the top of the train. The impact of the parameters of the viaduct structure, especially the viaduct's height, $H_{\text {viad }}$, on the HST channel can be observed through the Ricean $K$-factor and the distance between BS and MRS as follows. The timevarying distance between the BS and MRS is $D_{s}(t)=$ $\sqrt{\left(D_{\text {proj }}^{2}(t)+h^{2}\right)}$, where $h=H_{\mathrm{BS}}-\left(H_{\mathrm{viad}}+H_{\text {train }}+H_{\mathrm{MRS}}\right)$ and $D_{\text {proj }}(t)=\sqrt{\left(D_{\min }^{2}+D_{\text {ver }}^{2}(t)\right)}$. Moreover, $D_{\text {ver }}(t)=$ $\left(\sqrt{D_{\text {ver }}^{2}\left(t_{0}\right)+\left(v_{R} t\right)^{2}+2 D_{\text {ver }}\left(t_{0}\right) v_{R} t \cos \gamma_{R}}\right)$ and $D_{\min }=$ $L+W_{\text {viad }} / 2$. The Ricean $K$-factor, $K_{p q}(t)$, in viaduct scenario can be expressed as

$$
\begin{aligned}
& K_{p q}(t)(\mathrm{dB})= \\
& \left\{\begin{array}{l}
0.012 D_{s}(t)+0.29, D_{s}(t) \leq D_{B P} \\
\left(-0.00037 H_{\text {viad }}-\frac{0.18}{H_{\text {viad }}}+0.017\right) D_{s}(t) \\
+\left(0.148 H_{\text {viad }}+\frac{72}{H_{\text {viad }}}-1.71\right), D_{s}(t)>D_{B P} .
\end{array}\right.
\end{aligned}
$$

where $D_{B P}$ is the breakpoint distance that can be considered as the intercept distance used to remove the effect of the antenna.

\section{B. Cutting}

A sectional view of the cutting scenario with the main parameters of the cutting structure is illustrated in Fig. 3. The cutting structure, especially the sides, has a significant impact on the HST wireless propagation characteristics. As we explained earlier, the cutting scenario can be further classified into deep cutting $\left(H_{\text {cut }}>H_{\text {train }}+H_{\text {MRS }}\right)$ and low cutting $\left(H_{\text {cut }}<H_{\text {train }}+H_{\mathrm{MRS}}\right)$ scenarios. While the high altitude of the BS results in a dominant LoS component at the MRS side, the richness of the scatterers at the sides of the cutting will increase the possibility of multipath components and may lead to a severe fading. The impact of the parameters of the cutting structure, especially the cuttings's dimensions, $W_{\text {up }}$ and $W_{\text {down }}$ are represented by the Ricean $K$-factor and the distance between BS and MRS. The relative height between BS and MRS can be calculated as $h=H_{\text {cut }}+H_{\mathrm{BS}}-\left(H_{\text {train }}+H_{\text {MRS }}\right)$ while $D_{\text {min }}=L+W_{\text {up }} / 2$. The Ricean $K$-factor, $K_{p q}(t)$, in cutting scenario can be expressed as

$$
\begin{aligned}
& K_{p q}(t)(\mathrm{dB})= \\
& \left\{\begin{array}{l}
0.027 D_{s}(t)+0.41\left(W_{\text {up }}+W_{\text {down }}\right)-30.78, D_{s}(t) \leq D_{B P} \\
-0.0036 D_{s}(t)+0.41\left(W_{\text {up }}+W_{\text {down }}\right)-24.66, D_{s}(t)>D_{B P} .
\end{array}\right.
\end{aligned}
$$




$$
\mu_{R}^{(i)}(t)= \begin{cases}\gamma_{R}-\arccos \left(\frac{v_{R} t-\xi_{R}^{\left(n_{i}\right)}\left(t_{0}\right) \cos \left(\gamma_{R}-\mu_{R}^{(i)}\left(t_{0}\right)\right)}{\sqrt{\xi_{R}^{2\left(n_{i}\right)}\left(t_{0}\right)+\left(v_{R} t\right)^{2}-2 \xi_{R}^{\left(n_{i}\right)}\left(t_{0}\right) v_{R} t \cos \left(\gamma_{R}-\mu_{R}^{i}\left(t_{0}\right)\right)}}\right), & -\pi \leq \gamma_{R} \leq 0 \\ \gamma_{R}+\arccos \left(\frac{v_{R} t-\xi_{R}^{\left(n_{i}\right)}\left(t_{0}\right) \cos \left(\gamma_{R}-\mu_{R}^{(i)}\left(t_{0}\right)\right)}{\sqrt{\xi_{R}^{2\left(n_{i}\right)}\left(t_{0}\right)+\left(v_{R} t\right)^{2}-2 \xi_{R}^{\left(n_{i}\right)}\left(t_{0}\right) v_{R} t \cos \left(\gamma_{R}-\mu_{R}^{i}\left(t_{0}\right)\right)}}\right), & 0 \leq \gamma_{R} \leq \pi .\end{cases}
$$

Table II shows typical values of the different key scenariospecific parameters introduced earlier. These values are summarized based on a comprehensive survey of HST measurement campaigns in viaduct and cutting scenarios.

\section{Characterization of The HSt Channel Model}

In this Section, we will derive some of the statistical properties of the proposed generic non-stationary HST GBSM.

\section{A. Time-variant Autocorrelation Function (ACF)}

The normalized time-variant ACF can be derived as

$$
r(t, \Delta t)=\frac{\mathrm{E}\left\{h_{i, p q}(t) h_{i, p q}^{*}(t-\Delta t)\right\}}{\sqrt{\Omega_{i, p q} \Omega_{i, p^{\prime} q^{\prime}}}},
$$

where $(\cdot)^{*}$ denotes the complex conjugate operation and $\mathrm{E}\{\cdot\}$ designates the statistical expectation operator.

In the case of the LoS component,

$r^{\mathrm{LoS}}(t, \Delta t)=\frac{K_{p q}(t)}{1+K_{p q}(t)} e^{j 2 \pi f_{\max } \cos \left(\phi^{\mathrm{LoS}}(t-\Delta t)-\gamma_{R}\right) \Delta t}$.

In the case of the SB component,

$$
\begin{aligned}
r^{\mathrm{SB}_{i}}(t, \Delta t)=\frac{1}{N_{i}\left(1+K_{p q}(t)\right)} & \sum_{n_{i}=1}^{N_{i}} e^{j 2 \pi \xi_{T R}^{\left(n_{i}\right)}(t, \Delta t)} \\
& \times e^{j 2 \pi f_{\max } \cos \left(\phi_{R}^{\left(n_{i}\right)}-\gamma_{R}\right) \Delta t} .
\end{aligned}
$$

\section{B. Time-variant Level Crossing Rate (LCR)}

The LCR, $L(t, r)$, is by definition the average number of times per second that the signal envelope, $\left|h_{p q}(t)\right|$, crosses a specified level $r$ with positive / negative slope. Using the traditional PDF-based method [11], we derive the expression of LCR for HST channels as

$$
\begin{aligned}
& L(t, r)=\frac{2 r \sqrt{K_{p q}(t)+1}}{\pi^{3 / 2}} B(t) e^{-K_{p q}(t)-\left(K_{p q}(t)+1\right) r^{2}} \\
& \times \int_{0}^{\pi / 2} \cosh \left(2 \sqrt{K_{p q}(t)\left(K_{p q}(t)+1\right)} \cdot r \cos \theta\right) \\
& \times\left[e^{-(\chi(t) \sin \theta)^{2}}+\sqrt{\pi} \chi(t) \sin \theta \cdot \operatorname{erf}(\chi(t) \sin \theta)\right] d \theta,
\end{aligned}
$$

where $\cosh (\cdot)$ is the hyperbolic cosine function, $\operatorname{erf}(\cdot)$ is the error function, $B(t)=\sqrt{\frac{b_{2}(t)}{b_{0}(t)}-\frac{b_{1}^{2}(t)}{b_{0}^{2}(t)}}$, and $\chi(t)$ is equal to $\sqrt{\frac{K_{p q}(t) b_{1}^{2}(t)}{\left(b_{0}(t) b_{2}(t)-b_{1}^{2}(t)\right)}}$. Finally, parameters $b_{0}(t), b_{1}(t)$, and $b_{2}(t)$ are defined as

$$
b_{0}(t) \triangleq \mathrm{E}\left\{h_{p q}^{I}(t)^{2}\right\}=\mathrm{E}\left\{h_{p q}^{Q}(t)^{2}\right\},
$$

$$
\begin{gathered}
b_{1}(t) \triangleq \mathrm{E}\left\{h_{p q}^{I}(t) \dot{h}_{p q}^{Q}(t)\right\}=\mathrm{E}\left\{h_{p q}^{Q}(t) \dot{h}_{p q}^{I}(t)\right\}, \\
b_{2}(t) \triangleq \mathrm{E}\left\{\dot{h}_{p q}^{I}(t)^{2}\right\}=\mathrm{E}\left\{\dot{h}_{p q}^{Q}(t)^{2}\right\},
\end{gathered}
$$

where $h_{p q}^{I}(t)$ and $h_{p q}^{Q}(t)$ denote the in-phase and quadrature components of the complex fading envelope $h_{p q}(t)$, and $\dot{h}_{p q}^{I}(t)$ and $\dot{h}_{p q}^{Q}(t)$ denote the first derivative of $h_{p q}^{I}(t)$ and $h_{p q}^{Q}(t)$, respectively.

Using (1) - (3), the parameters $b_{0}(t), b_{1}(t)$, and $b_{2}(t)$ can be calculated as

$$
\begin{gathered}
b_{0}(t)=\frac{1}{K_{p q}(t)+1}, \\
b_{1}(t)=\frac{b_{0}(t)}{N_{i}} \sum_{n_{i}=1}^{N_{i}} f_{\max } \cos \left(\phi_{R}^{\left(n_{i}\right)}-\gamma_{R}\right), \\
b_{2}(t)=\frac{b_{0}(t)}{N_{i}} \sum_{n_{i}=1}^{N_{i}}\left[f_{\max } \cos \left(\phi_{R}^{\left(n_{i}\right)}-\gamma_{R}\right)\right]^{2} .
\end{gathered}
$$

\section{Stationary Interval / Stationary Distance}

The stationary interval can be calculated using averaged power delay profiles (APDPs) that can be expressed as [10]

$$
\overline{P_{h}}\left(t_{k}, \tau\right)=\frac{1}{N_{P D P}} \sum_{k}^{k+N_{P D P}-1}\left|h_{p q}\left(t_{k}, \tau\right)\right|^{2}
$$

where $N_{P D P}$ is the number of power delay profiles to be averaged, $t_{k}$ is the time of the $k$-th drop (snapshot), and $h_{p q}\left(t_{k}, \tau\right)=\sum_{i=1}^{I} h_{i, p q}\left(t_{k}\right) \delta\left(\tau-\tau_{i}\right)$. The correlation coefficient between two APDPs can be calculated as

$$
c\left(t_{k}, \Delta t\right)=\frac{\int \overline{P_{h}}\left(t_{k}, \tau\right) \overline{P_{h}}\left(t_{k}+\Delta t, \tau\right) \mathrm{d} \tau}{\max \left\{\int \overline{P_{h}}\left(t_{k}, \tau\right)^{2} \mathrm{~d} \tau, \int \overline{P_{h}}\left(t_{k}+\Delta t, \tau\right)^{2} \mathrm{~d} \tau\right\}} .
$$

The stationary interval can be then calculated as

$$
T_{s}\left(t_{k}\right)=\max \left\{\left.\Delta t\right|_{c\left(t_{k}, \Delta t\right) \geq c_{\text {thresh }}}\right\},
$$

where $c_{\text {thresh }}$ is a given threshold of the correlation coefficient.

Analogously, the stationary distance can be calculated as

$$
S D\left(t_{k}\right)=\max \left\{\left.\Delta D\right|_{c\left(t_{k}, \Delta t\right) \geq c_{\text {thresh }}}\right\},
$$

where $\Delta D=v_{R} \times \Delta t$.

\section{Numerical Results And Analysis}

In this section, we will verify the theoretical results of the derived statistical properties in Section IV using relevant measurement data from the literature. 


\section{A. Viaduct}

Here, we compare the derived ACF and LCR with the ones of the measured HST channel in viaduct scenario reported in [12]. Unless specified otherwise, the parameters for our analysis, obtained from the measurement campaign in [12], are: $f_{c}=930 \mathrm{MHz}, v_{R}=180 \mathrm{~km} / \mathrm{h}, L=15 \mathrm{~m}, H_{\text {viad }}=20 \mathrm{~m}$, $H_{\mathrm{BS}}=44 \mathrm{~m}, H_{\mathrm{MRS}}=30 \mathrm{~cm}, H_{\text {train }}=3.8 \mathrm{~m}$, cellular radius $=2502 \mathrm{~m}$, therefore, $D_{s}(t) \in[0,2502) \mathrm{m}, a_{1}=\frac{D_{s}}{2}+150 \mathrm{~m}$, $D_{B P}=400 \mathrm{~m}, \gamma_{R}=0$, and $N=50$. Fig. 4 shows the absolute values of the ACFs of the proposed generic HST model and the measured ACF taken from Fig. 9 (Case 3) in [12]. Since the measurement has been conducted under GSM$\mathrm{R}$ system, the measured channel is a narrowband one and hence we used $h_{p q}(t, \tau)=h_{1, p q}(t) \delta\left(\tau-\tau_{1}\right)$ where $h_{1, p q}(t)$ is given in (1). It is noteworthy that the ACFs in Fig. 4 are plotted as functions of distance $\Delta D=v_{R} \times \Delta t$ for comparison purposes.

Fig. 5 shows the LCR of the measured channel, obtained from Fig. 8 in [12], and the derived LCRs of the proposed generic HST channel model. The very good agreement between the proposed generic model and the measurement data demonstrates the utility of our HST generic channel model in viaduct scenarios.

\section{B. Cutting}

We use the measurement in [13] to verify the applicability of the proposed generic model in cutting scenario. The simulation parameters, obtained from the measurement campaign reported in [13], are listed here or specified otherwise: $f_{c}=930 \mathrm{MHz}, v_{R}=260 \mathrm{~km} / \mathrm{h}, L=15 \mathrm{~m}, H_{\text {cut }}=7.1 \mathrm{~m}$, $H_{\mathrm{BS}}=28 \mathrm{~m}, H_{\mathrm{MRS}}=30 \mathrm{~cm}, H_{\text {train }}=3.8 \mathrm{~m}, W_{\text {up }}=53.93 \mathrm{~m}$, $W_{\text {down }}=17.78 \mathrm{~m}$, cellular radius $=1410 \mathrm{~m}$, therefore, $D_{s}(t) \in[0,1410) \mathrm{m}, a_{1}=\frac{D_{s}}{2}+150 \mathrm{~m}, D_{B P}=200 \mathrm{~m}$, $\gamma_{R}=0$, and $N=50$.

Fig. 6 shows the absolute values of the ACFs $r(t, \Delta t)$ of the proposed generic HST model using different cutting's dimensions. Two cuttings scenarios from [13] are considered, i.e., Cutting $1\left(W_{\text {up }}=53.93 \mathrm{~m} \mathrm{\&} W_{\text {down }}=14.78 \mathrm{~m}\right)$ and Cutting $2\left(W_{\text {up }}=58.30 \mathrm{~m} \& W_{\text {down }}=15.16 \mathrm{~m}\right)$. A higher correlation in Cutting 2 in comparison with Cutting 1 can be noticed. This can be explained based on the difference in the cuttings' dimensions. Since Cutting 2 is wider than Cutting 1 , a stronger LoS component represented by a higher $K$ factor value will be observed at the receiver according to III-B which in turn will result in higher ACF values. Fig. 7 shows the normalized LCR of the measured channel, obtained from Fig. 9(a) in [13], and the derived LCRs of the proposed generic HST channel model. The simulation results of the HST channel model and the measured channel match very well.

Finally, Fig. 8 shows the stationary distances of the generic HST channel model in viaduct and cutting scenarios based on the measurement setup parameters reported in [14]. The simulation parameters, obtained from the aforementioned measurement campaign [14], are: viaduct $\left(f_{c}=1.89 \mathrm{GHz}\right.$, $v_{R}=285 \mathrm{~km} / \mathrm{h}, L=15 \mathrm{~m}, H_{\mathrm{viad}}=20 \mathrm{~m}, H_{\mathrm{BS}}=20 \mathrm{~m}$, $\left.H_{\text {MRS }}=30 \mathrm{~cm}, H_{\text {train }}=3.8 \mathrm{~m}\right)$; cutting: $\left(f_{c}=2.35 \mathrm{GHz}\right.$, $v_{R}=200 \mathrm{~km} / \mathrm{h}, L=20 \mathrm{~m}, H_{\text {cut }}=5 \mathrm{~m}, H_{\mathrm{BS}}=28 \mathrm{~m}$, $H_{\text {MRS }}=30 \mathrm{~cm}, H_{\text {train }}=3.8 \mathrm{~m}, W_{\text {up }}=40 \mathrm{~m}, W_{\text {down }}=16$ $\mathrm{m})$. The mean values of the stationary distances of the viaduct and cutting scenarios are $3.26 \mathrm{~m}$ and $2.86 \mathrm{~m}$, respectively.
This agrees with the range of the measured averaged stationary intervals that is reported in [14] as $2.8 \mathrm{~m}-4.2 \mathrm{~m}$.

\section{CONCLUSIONS}

In this paper, a novel generic HST GSBM is proposed for different HST scenarios. This generic model takes into account the impact of different scenario-specific parameters of each HST scenario on the wireless channel, e.g., the height of the viaduct and the dimensions of the cuttings. Verified by real measurement data from the literature, the proposed model can be applied on three of the most common HST scenarios, i.e., open space, viaduct, and cutting.

\section{ACKNOWLEDGEMENTS}

The authors gratefully acknowledge the support of this work from the EU FP7 QUICK project under Grant PIRSES-GA-2013-612652, the EU H2020 5G Wireless project under Grant 641985, the Ministry of Science and Technology of China through the 863 Project in 5G under Grant 2014AA01A707, the National Science and Technology Major Project under grant 2014ZX03003012-001, and 973 project under grant 2012CB316100.

\section{REFERENCES}

[1] C.-X. Wang, F. Haider, X. Gao, X.-H. You, Y. Yang, D. Yuan, H. Aggoune, H. Haas, S. Fletcher, E. Hepsaydir, "Cellular architecture and key technologies for 5G wireless communication networks," IEEE Commun. Mag., vol. 52, no. 2, pp. 122-130, Feb. 2014.

[2] C.-X. Wang, A. Ghazal, B. Ai, P. Fan, and Y. Liu, "Channel measurements and models for high-speed train communication systems: a survey," IEEE Commun. Surveys Tuts, submitted for publication.

[3] B. Ai, X. Cheng, T. Kurner, Z.-D. Zhong, K. Guan, R.-S. He, L. Xiong D. W. Matolak, D. G. Michelson, and C. Briso-Rodriguez, "Challenges toward wireless communications for high-speed railway," IEEE Trans. Intell. Transp. Syst., vol. 15, no. 5, pp. 2143-2158, Oct. 2014.

[4] B. Ai, R. He, Z. Zhong, K. Guan, B. Chen, P. Liu, and Y. Li, "Radio wave propagation scene partitioning for high-speed rails," Int. J. Antennas Propag., vol. 2012, Article ID 815232, 7 pages, 2012.

[5] P. Kyösti et al., "WINNER II channel models," IST-4-027756, WINNER II D1.1.2, v1.2, Apr. 2008.

[6] ITU-R M.2135-1, "Guidelines for Evaluation of Radio Interface Technologies for IMT-Advanced," Geneva, Switzerland, Rep. ITU-R M.21351, Dec. 2009.

[7] A. Ghazal, C.-X. Wang, B. Ai, D. Yuan, and H. Haas, "A nonstationary wideband MIMO channel model for high-mobility intelligent transportation systems," IEEE Trans. Intell. Transp. Syst., vol. 16, no. 2, pp. 885-897, Apr. 2015.

[8] R. He, D. Zhong, B. Ai, and C. Oestges, "Shadow fading correlation in high-speed railway environments," IEEE Trans. Veh. Technol., vol. 64, no. 7, pp. 2762-2772, July 2015.

[9] X. Cheng, C.-X. Wang, D. I. Laurenson, S. Salous, and A. V. Vasilakos, "An adaptive geometry-based stochastic model for non-isotropic MIMO mobile-to-mobile channels," IEEE Trans. Wireless Commun., vol. 8, no. 9, pp. 4824-4835, Sept. 2009.

[10] B. Chen, Z. Zhong, and B. Ai, "Stationarity intervals of time-variant channel in high speed railway scenario," J. China Commun., vol. 9, no. 8, pp. 64-70, Aug. 2012.

[11] N. Youssef, C.-X. Wang, and M. Pätzold, "A study on the second order statistics of Nakagami-Hoyt mobile fading channels," IEEE Trans. Veh. Technol., vol. 54, no. 4, pp. 1259-1265, July 2005.

[12] R. He et al., "Measurements and analysis of propagation channels in high-speed railway viaducts," IEEE Trans. Wireless Commun., vol. 12, no. 2, pp. 794-805, Feb. 2013.

[13] R. He et al., "Short-term fading behavior in high-speed railway cutting scenario: measurements, analysis, and statistical models," IEEE Trans. Antennas Propag., vol. 61, no. 4, pp. 2209-2222, Apr. 2013. 
TABLE I. DEFINITION OF PARAMETERS IN FIG. 1.

\begin{tabular}{|c|l|}
\hline \multicolumn{1}{|c|}{ Parameters } & Definition \\
\hline$D_{s}(t)$ & distance between the BS and MRS \\
\hline$f_{s}(t)$ & half length of the distance between the two foci of ellipses \\
\hline$a_{i}(t), b_{i}(t)$ & semi-major axis and semi-minor axis of the $i$ th ellipse, respectively \\
\hline$v_{R}, \gamma_{R}$ & MRS speed and angle of motion, respectively \\
\hline$\Delta x_{T}, \Delta x_{R}$ & antenna element spacings of the BS and MRS, respectively \\
\hline$\beta_{T}, \beta_{R}$ & tilt angles of the BS and MRS antenna arrays in the x-y plane (relative to the x-axis), respectively \\
\hline$\phi_{T}^{\text {LoS }}(t), \phi_{R}^{\left(n_{i}\right)}(t)$ & AoA of the LoS path and AoA of the wave travelling from an effective scatterer $s^{\left(n_{i}\right)}$ to the MRS, respectively \\
\hline$\phi_{T}^{\left(n_{i}\right)}(t)$ & AoD of the wave that impinges on the effective scatterer $s^{\left(n_{i}\right)}$ \\
\hline$\xi, \xi_{T}^{\left(n_{i}\right)}(t)$, and $\xi_{R}^{\left(n_{i}\right)}(t)$ & distances $d\left(T_{p}\right.$, MRS), $d\left(\mathrm{BS}, s^{\left(n_{i}\right)}\right)$, and $d\left(s^{\left(n_{i}\right)}\right.$, MRS $)$, respectively \\
\hline$\varepsilon_{p q}, \varepsilon_{p n_{i}}, \varepsilon_{n_{i} q}$ & distances $d\left(T_{p}, R_{q}\right), d\left(T_{p}, s^{\left(n_{i}\right)}\right)$, and $d\left(s^{\left(n_{i}\right)}, R_{q}\right)$, respectively \\
\hline
\end{tabular}

TABLE II. TyPiCAL VALUES OF PARAMETERS IN FigS. $2 \& 3$.

\begin{tabular}{|c|l|c|l|}
\hline Viaduct & Values & Cutting & Values \\
\hline$H_{\text {viad }}$ & $10-30 \mathrm{~m}$ & $H_{\text {cut }}$ & $2-10 \mathrm{~m}$ \\
\hline$H_{\mathrm{BS}}$ & $H_{\text {viad }}+(20-30) \mathrm{m}$ & $H_{\mathrm{BS}}$ & $20-30 \mathrm{~m}$ \\
\hline$H_{\text {MRS }}$ & $30 \mathrm{~cm}$ & $H_{\text {MRS }}$ & $30 \mathrm{~cm}$ \\
\hline$H_{\text {train }}$ & $3.8 \mathrm{~m}$ & $H_{\text {train }}$ & $3.8 \mathrm{~m}$ \\
\hline$L$ & $10-30 \mathrm{~m}$ & $L$ & $10-30 \mathrm{~m}$ \\
\hline$W_{\text {viad }}$ & $10-20 \mathrm{~m}$ & $W_{\text {down }}$ & $14-20 \mathrm{~m}$ \\
\hline & & $W_{\text {up }}$ & $45-65 \mathrm{~m}$ \\
\hline
\end{tabular}
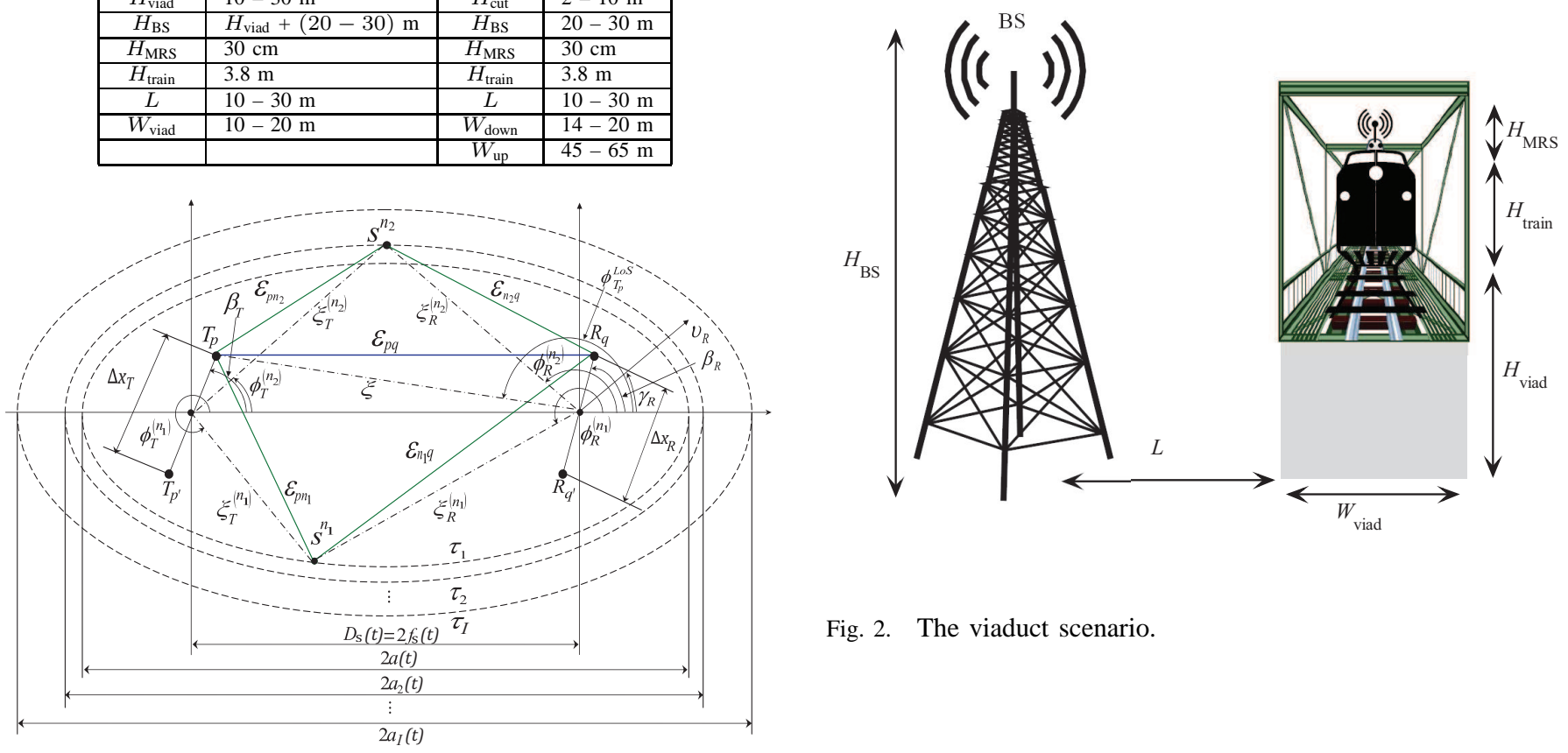

Fig. 2. The viaduct scenario.

Fig. 1. The GBSM for a wideband MIMO HST channel.

[14] High speed railway channel characterstics, draft revision to Recommendation ITU-R P.1411-7, Document 3K/143-E, Apr. 2015.

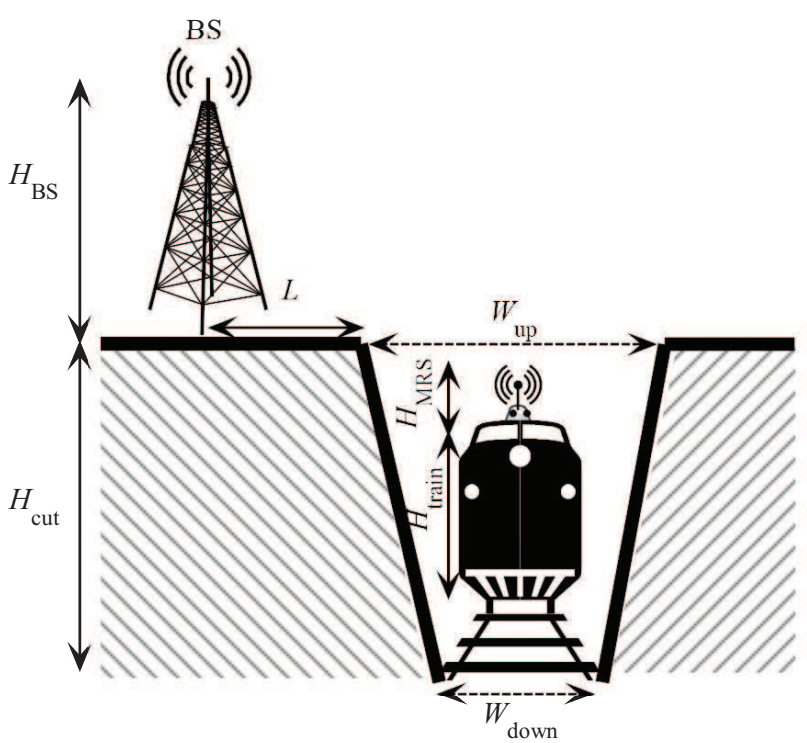

Fig. 3. The cutting scenario. 


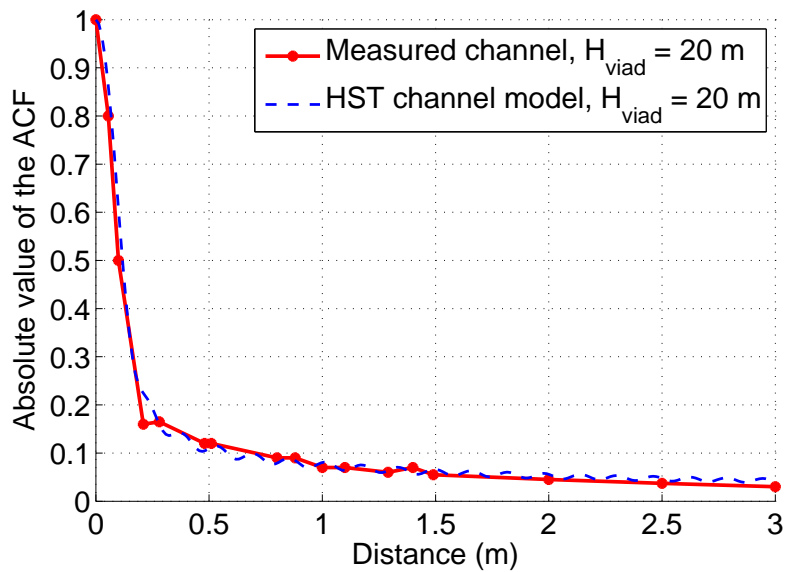

Fig. 4. The absolute values of the time-variant ACFs of the generic HST channel model and measured channel in viaduct scenario [12] $\left(f_{c}=930 \mathrm{MHz}, v_{R}=180 \mathrm{~km} / \mathrm{h}, L=15 \mathrm{~m}, H_{\text {viad }}=20 \mathrm{~m}\right.$, $H_{\mathrm{BS}}=44 \mathrm{~m}, H_{\mathrm{MRS}}=30 \mathrm{~cm}, H_{\text {train }}=3.8 \mathrm{~m}, D_{B P}=400 \mathrm{~m}$, $\left.\gamma_{R}=0, \beta_{R}=\beta_{T}=45^{\circ}, \mu_{R}^{(i)}\left(t_{0}\right)=63^{\circ}, k_{R}^{(i)}=2.7, N=50\right)$.

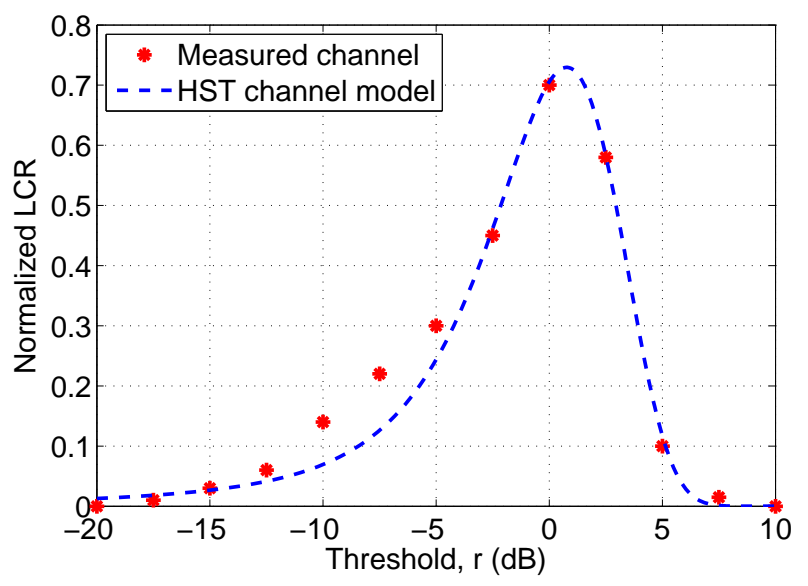

Fig. 5. The LCRs of the generic HST channel model and the measured channel in [12] in viaduct scenario $\left(f_{c}=930 \mathrm{MHz}, v_{R}=180 \mathrm{~km} / \mathrm{h}\right.$, $L=15 \mathrm{~m}, H_{\text {viad }}=20 \mathrm{~m}, H_{\mathrm{BS}}=44 \mathrm{~m}, H_{\mathrm{MRS}}=30 \mathrm{~cm}$, $H_{\text {train }}=3.8 \mathrm{~m}, D_{B P}=400 \mathrm{~m}, \gamma_{R}=0, \beta_{R}=\beta_{T}=60^{\circ}$, $\left.\mu_{R}^{(i)}\left(t_{0}\right)=85^{\circ}, k_{R}^{(i)}=7.3, N=50\right)$.

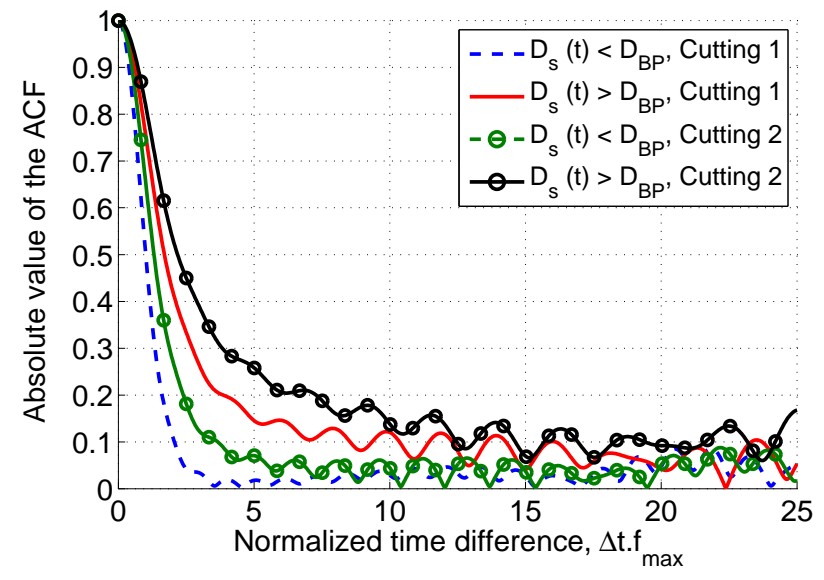

Fig. 6. The absolute values of the time-variant ACFs of the generic HST channel model for different cuttings' dimensions [13] (Cutting 1: $W_{\text {up }}=53.93 \mathrm{~m} \& W_{\text {down }}=14.78 \mathrm{~m}$, Cutting $2: W_{\text {up }}=58.30 \mathrm{~m}$ $\& W_{\text {down }}=15.16 \mathrm{~m}, f_{c}=930 \mathrm{MHz}, v_{R}=260 \mathrm{~km} / \mathrm{h}, L=15 \mathrm{~m}$, $H_{\text {cut }}=7.1 \mathrm{~m}, H_{\mathrm{BS}}=28 \mathrm{~m}, H_{\mathrm{MRS}}=30 \mathrm{~cm}, H_{\text {train }}=3.8 \mathrm{~m}$, $D_{B P}=200 \mathrm{~m}, \gamma_{R}=0, \beta_{R}=\beta_{T}=45^{\circ}, \mu_{R}^{(i)}\left(t_{0}\right)=63^{\circ}$, $k_{R}^{(i)}=15, N=50$ ).

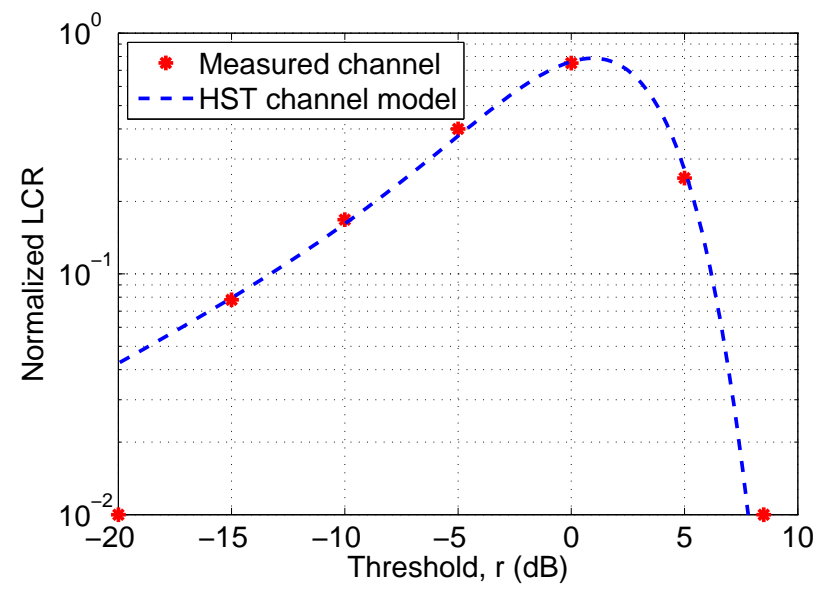

Fig. 7. The LCRs of the generic HST channel model and the measured channel in [13] in cutting scenario $\left(f_{c}=930 \mathrm{MHz}, v_{R}=260 \mathrm{~km} / \mathrm{h}\right.$, $L=15 \mathrm{~m}, H_{\text {cut }}=7.1 \mathrm{~m}, H_{\mathrm{BS}}=28 \mathrm{~m}, H_{\mathrm{MRS}}=30 \mathrm{~cm}$, $H_{\text {train }}=3.8 \mathrm{~m}, W_{\text {up }}=53.93 \mathrm{~m}, W_{\text {down }}=14.78 \mathrm{~m}, D_{B P}=200 \mathrm{~m}$, $\left.\gamma_{R}=0, \beta_{R}=\beta_{T}=60^{\circ}, \mu_{R}^{(i)}\left(t_{0}\right)=145^{\circ}, k_{R}^{(i)}=4, N=50\right)$. 


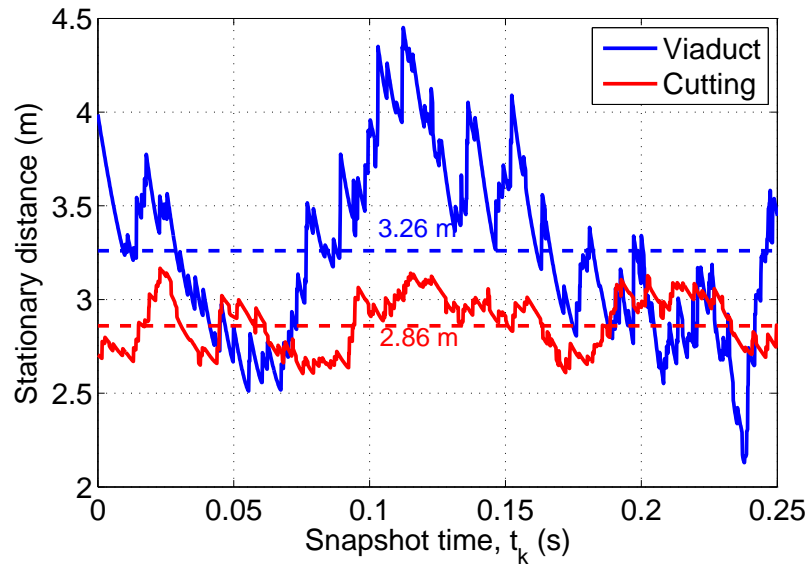

Fig. 8. The stationary distances of the generic HST channel model in viaduct and cutting scenarios (viaduct: $f_{c}=1.890 \mathrm{GHz}$, $v_{R}=285 \mathrm{~km} / \mathrm{h}, L=15 \mathrm{~m}, H_{\text {viad }}=20 \mathrm{~m}, H_{\mathrm{BS}}=20 \mathrm{~m}$, $H_{\mathrm{MRS}}=30 \mathrm{~cm}, H_{\text {train }}=3.8 \mathrm{~m}$, cutting: $f_{c}=2.35 \mathrm{GHz}$, $v_{R}=200 \mathrm{~km} / \mathrm{h}, L=20 \mathrm{~m}, H_{\text {cut }}=5 \mathrm{~m}, H_{\mathrm{BS}}=28 \mathrm{~m}$, $\left.H_{\text {MRS }}=30 \mathrm{~cm}, H_{\text {train }}=3.8 \mathrm{~m}, W_{\text {up }}=40 \mathrm{~m}, W_{\text {down }}=16 \mathrm{~m}\right)$. 